\title{
Rehabilitación Cognitiva en los Trastornos Psiquiátricos
}

\section{Cognitive Rehabilitation in Psychiatric Disorders}

Luis Felipe Matovelle Romero *1, Jorge Fabián Guapisaca Juca ${ }^{2}$, Gladys Alexandra Bueno Pacheco ${ }^{3}$, Daniela Margarita Tripaldi Proaño ${ }^{4}$,

INFORMACIÓN DEL

ARTÍCULO

Fecha de recepción: junio 26, 2020.

Fecha de aceptación: agosto 20, 2020

$\bar{l}$ Universidad del Azuay. Maestría en Neuropsicología e investigación.

E-mail: luch@es.uazuay.edu.ec

E-mail: luch@es.
Código ORCID:

https://orcid.org/0000-0002-5553-3272

${ }^{2}$ Universidad del Azuay. Geriatra por la Universidad de Santiago de Chile.

E-mail: fguapisaca@azuay.edu.ec

Código ORCID

https://orcid.org/0000-0003-2124-818X

3 Universidad del Azuay Magister en Diagnostico y Rehabilitación Neuropsicológica por la Benemérita Universidad Autónoma de Puebla.

E-mail: abueno@uazuay.edu.ec

Código ORCID

https://orcid.org/0000-0001-7188-1210

4 Doctor en Psicología CognitivaExistencial. Healthy Mind Works (Países Bajos), docente invitada de la Maestría en Neuropsicología de la Universidad del Azuay (Ecuador).

E-mail: danielatripaldi@gmail.com

Código ORCID:

https://orcid.org/0000-0001-5615-1914

CITACIÓN: Matovelle Romero, L.F., Guapisaca, J.F., Bueno, G.A. \& Merchán, P.G. (2021). Rehabilitación cognitiva en los Trastornos Psiquiátricos. Investigatio, 16, 8-21

doi:10.31095/ investigatio.2021.16.2

ENLACE DOI:

http://dx.doi.org/10.31095/investigatio 2021.16.2

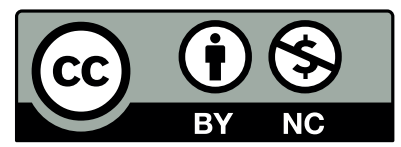

\section{Resumen}

Los trastornos cognitivos dentro de los trastornos psiquiátricos son considerados un problema a nivel global; quienes lo padecen evidencian un marcado deterioro en prácticamente todas las áreas cognitivas, en consecuencia, la rehabilitación cognitiva se vuelve crucial para potencializar su capacidad funcional y su ajuste en el medio social. El objetivo principal de esta investigación es explorar los avances en aplicación de procedimientos de rehabilitación cognitiva en trastornos psiquiátricos, mediante una revisión exhaustiva de la literatura internacional reciente, encontrada dentro de las bases de datos Scopus y Web of Science. De dicha recopilación se seleccionaron 18 artículos, referentes a estudios empíricos. Se concluye que la rehabilitación cognitiva es efectiva al momento de la intervención en pacientes con trastornos psiquiátricos, se destaca la utilización generalizada de softwares específicos para la intervención, así como la presencia de modelos de intervención que integran otros tratamientos.

\section{Palabras Clave:}

Rehabilitación Cognitiva, Trastornos Psiquiátricos, Estudios Empíricos Trastornos cognitivos, Neuropsicología, Revisión de literatura.

Clasificación JEL: I10.

\begin{abstract}
Cognitive disorders within psychiatric pathologies are considered a problem at a global scale; those who suffer from them show a marked deterioration in a wide range of cognitive areas. Consequently, cognitive rehabilitation becomes crucial to enhance their functional capacity and their adjustment in the social environment. The main objective of this research is to explore the advances in the application of cognitive rehabilitation procedures in psychiatric disorders, through an exhaustive review of recent international literature, found within the Scopus and Web of Science databases. Eighteen articles, referring to empirical studies, were obtained from this compilation. It is concluded that cognitive rehabilitation is effective at the time of intervention in patients with psychiatric disorders. The generalized use of specific software for the intervention is highlighted, as well as the presence of intervention models that integrate other treatments.
\end{abstract}

\section{Keywords:}

Cognitive rehabilitation, Psychiatric disorders, Empirical studies, Cognitive disorders, Neuropsychology, Literature review.

JEL Classification: I10.

$$
8
$$

\footnotetext{
INVESTIGATIO No. 16, marzo 2021, pp. 8-2

(C) Universidad Espíritu Santo - UEES
}

ISSN: 1390 - 6399・ISSN-e: 2602 - 8336 


\section{Introducción}

Las dificultades cognitivas asociadas a trastornos psiquiátricos conforman un importante problema de salud a nivel mundial; se ha evidenciado que quienes los padecen pueden vivenciar un marcado deterioro en prácticamente todas las áreas cognitivas (Moreno, García, Gámez y Moreno, 2016). Frente a esto, los tratamientos psicoterapéuticos comunes y tratamientos farmacológicos por sí solos no logran establecer evidencia consistente sobre su mejoría, por ello, la rehabilitación cognitiva se vuelve crucial para potencializar su capacidad funcional y su ajuste en el medio social (Ojeda et al., 2012).

Sutu, Moro y Mezquita (2015), mencionan que aproximadamente el $40 \%$ de pacientes que presentan patologías psiquiátricas pueden manifestar alteraciones cognitivas moderadas $\mathrm{o}$ graves. Así, por ejemplo, un indicador común entre pacientes con trastorno bipolar, cuadros depresivos, trastornos psicóticos, entre otros, suelen ser las quejas sobre su funcionamiento general, mostrando una marcada deficiencia dentro de su rendimiento cognitivo (Cardoner y Vicent, 2017).

La disfuncionalidad cognitiva se manifiesta mediante déficits en los procesos de atención, procesamiento de la información, memoria de trabajo, estados de alerta, entre otros (Sánchez, 2012). En este sentido, se puede identificar dos funciones que se encuentran deficitarias en los pacientes con patologías psiquiátricas; las funciones cognitivas básicas que comprenden la atención, memoria, memoria de trabajo, capacidad de abstracción, lenguaje y funciones motoras; y las funciones de cognición social como la correcta percepción del entorno, resolución de conflictos, capacidad de prever las reacciones de los demás, etc. (Casas, Escandell, Ribas y Ochoa, 2010). Inclusive, Garcelan (2015) manifiesta que, es probable que las deficiencias dentro de las funciones cognitivas básicas sean el mayor motivo de las dificultades a nivel de la esfera social y familiar.

Cuesta et al., (2015), muestran evidencia frente a que las alteraciones cognitivas se manifiestan inclusive antes del diagnóstico psiquiátrico, y que, por lo general, tienden a mantenerse invariables; no obstante, con el paso de los años y las recaídas suelen potencializarse. Esto permite comprender que, aunque los déficits cognitivos se exacerban en la fase activa de varios trastornos, su presencia e implicaciones se mantienen inclusive al alcanzar diversos niveles de recuperación, de aquí la importancia de establecer procedimientos rehabilitadores que potencialicen la capacidad del individuo en su totalidad bio-psico-social (Perona, 2003). De esta manera, una intervención temprana de rehabilitación cognitiva en estos trastornos juega un rol fundamental, pues permitiría recuperar su funcionalidad, independencia, y mejorar su calidad de vida significativamente (Cuesta et al, 2018; Garcelan, 2015).

Con base en lo mencionado 
anteriormente, el objetivo principal de esta revisión es indagar acerca de los avances en aplicación de procedimientos de rehabilitación cognitiva en trastornos psiquiátricos, mediante una revisión exhaustiva de la literatura internacional reciente, haciendo hincapié en aquellos que utilicen técnicas de corte neuropsicológico.

\section{Metodología}

Para establecer el diseño de investigación se utilizaron los lineamientos del Preferred Reporting Items for Systematic Reviews and Meta-Analyses (PRISMA) el cual facilita la recolección, procesamiento y presentación en revisiones de la literatura (Moher, Liberati, Tetzlaff, Altman, 2009). Se efectúo una revisión crítica de la bibliografía de investigaciones publicadas en las bases de datos Web of Science y Scopus sobre la temática de rehabilitación cognitiva en los trastornos psiquiátricos. La selección de artículos se realizó en función de los criterios de inclusión y exclusión que se exponen en la tabla 1 .

Para realizar la búsqueda se utilizó las siguientes palabras clave: "cognitive rehabilitation", "mental disorder". En un primer momento, los resultados en las bases de datos de Web of Science y Scopus mostraron 856 y 2958 artículos respectivamente, para un total de 3814 artículos sobre esta temática. Posteriormente se procedió a filtrar los resultados en función de un límite temporal de 5 años (2015-2020), del mismo modo se aplicó un filtro de búsqueda en función del área de conocimiento: neurociencias y psicología, lo cual permitió delimitar investigaciones al tema planteado (72 en Web of Science y 123 en Scopus, para un total de 195).

Subsecuentemente se procedió a realizar una elección manual en donde se excluyeron los artículos que no cumplían con los criterios de inclusión mediante una lectura crítica de título, resumen y población de estudio, además se complementó la búsqueda mediante la modalidad bola de nieve con la finalidad de localizar estudios no hallados en primera instancia. Del total de 65 referencias obtenidas tras los criterios de filtro, 23 en Web of Science y 42 en Scopus, se seleccionaron 18 documentos

Tabla 1 .

Criterios de inclusión y exclusión

\begin{tabular}{ll}
\hline \multicolumn{1}{c}{ Criterios de inclusión } & \multicolumn{1}{c}{ Criterios de exclusión } \\
\hline Investigaciones empíricas & Investigaciones diferentes al idioma \\
Muestra de población adulta & inglés y español \\
Investigaciones asociadas a trastornos psiquiátricos & Revisiones de literatura no \\
Con fecha entre 2015-2020 & sistematizadas \\
Investigaciones pertenecientes al área de estudio de la & Capítulos de libros y entrevistas a \\
psicología y neurociencias & profesionales \\
& Estudios de caso simple \\
& Tesis o investigaciones de pre-grado \\
\hline
\end{tabular}

Fuente: Elaboración propia. 
de interés, 10 en Web of Sicence y 8 en Scopus. (Ver tabla 2).

\section{Resultados}

Para la presentación de resultados obtenidos en el análisis de cada uno de los estudios relacionados al objetivo de investigación, se agruparon los estudios alrededor del diagnóstico identificado, siendo 11 relacionados al espectro de la esquizofrenia, 3 a trastornos neurocognitivos, 3 a trastornos por consumo y un único estudio sobre trastornos de la personalidad, este apartado se desarrolla a partir de tal agrupación, para un análisis detallado se tomó en cuenta la cantidad de muestra, las funciones evaluadas, las herramientas utilizadas, los procesos de intervención y los resultados más relevantes. Los resultados se muestran en detalle en la Tabla 3.

En las investigaciones encontradas, 11 de ellas estaban centradas en el

Tabla 2.

Registro de búsquedas realizadas

\begin{tabular}{|c|c|c|c|c|c|c|c|}
\hline $\begin{array}{c}\text { Base de } \\
\text { datos }\end{array}$ & $\begin{array}{c}\text { Palabras clave } \\
\text { usadas en la } \\
\text { búsqueda }\end{array}$ & $\begin{array}{c}\text { Registros } \\
\text { encontrados }\end{array}$ & $\begin{array}{l}\text { Últimos } \\
5 \text { años }\end{array}$ & $\begin{array}{c}\text { Área de } \\
\text { estudio } \\
\text { neurociencias }\end{array}$ & $\begin{array}{l}\text { Lectura } \\
\text { de título } \\
\text { y } \\
\text { resumen }\end{array}$ & $\begin{array}{c}\text { Bola } \\
\text { de } \\
\text { Nieve }\end{array}$ & Usados \\
\hline $\begin{array}{l}\text { Web of } \\
\text { Science }\end{array}$ & $\begin{array}{c}\text { "Cognitive } \\
\text { rehabilitation"; } \\
\text { "mental } \\
\text { disorder" }\end{array}$ & 856 & 370 & 72 & 23 & 2 & 8 \\
\hline Scopus & $\begin{array}{c}\text { "Cognitive } \\
\text { rehabilitation"; } \\
\text { "mental } \\
\text { disorder" }\end{array}$ & 2958 & 967 & 123 & 42 & 1 & 10 \\
\hline Total & & 3814 & 1337 & 195 & 65 & 3 & 18 \\
\hline
\end{tabular}

Fuente: Elaboración propia. 
Tabla 3.

Resultados

\begin{tabular}{|c|c|c|c|c|}
\hline Autores & $\begin{array}{l}\text { Trastorno y } \\
\text { muestra* }\end{array}$ & $\begin{array}{c}\text { Función abordada } \\
\text { y herramientas }\end{array}$ & $\begin{array}{l}\text { Intervención } \\
\text { y tiempo }\end{array}$ & $\begin{array}{l}\text { Resultado } \\
\text { Relevante }\end{array}$ \\
\hline $\begin{array}{l}\text { Rodríguez, } \\
\text { Caballero, } \\
\text { González y } \\
\text { Cartaya, } \\
2019\end{array}$ & $\begin{array}{l}\text { Esquizofre } \\
\text { nia, } \\
\text { Trastorno } \\
\text { bipolar } \\
\\
47 \\
\text { pacientes }\end{array}$ & $\begin{array}{l}\text { Funciones ejecutivas, } \\
\text { aprendizaje verbal y } \\
\text { memoria. } \\
\text { Herramientas de } \\
\text { funciones: MEC35, } \\
\text { DSMT, WAIS-R, } \\
\text { TMT, Velocidad de } \\
\text { procesamiento DSST. }\end{array}$ & $\begin{array}{l}\text {-Remediación } \\
\text { cognitiva mediante } \\
\text { sotfware Cogpack } \\
\text { personalizado de } \\
\text { acuerdo con } \\
\text { evaluación previa. } \\
\text {-Apoyo individual. } \\
32 \text { sesiones }\end{array}$ & $\begin{array}{l}\text { Los participantes mejoraron más } \\
\text { que el grupo control durante el } \\
\text { período de seguimiento en las } \\
\text { medidas de funcionamiento } \\
\text { cognitivo (significativamente } \\
\text { mayor en funciones ejecutivas, } \\
\text { aprendizaje verbal y memoria) y } \\
\text { obtuvieron porcentajes de } \\
\text { empleo más altos durante el } \\
\text { período de seguimiento. }\end{array}$ \\
\hline $\begin{array}{c}\text { Corrivetti, } \\
\text { Caserta, } \\
\text { Perna, } \\
2018\end{array}$ & $\begin{array}{l}\text { Esquizofre } \\
\text { nia } \\
\\
47 \\
\text { pacientes }\end{array}$ & $\begin{array}{l}\text { Atención, memoria verbal, } \\
\text { funciones ejecutivas, } \\
\text { coordinación y velocidad } \\
\text { psicomotora. } \\
\text { Clínicas: PANSS, MMPI-2 } \\
\text { Neuropsicológicas: } \\
\text { WAIS-R, BACS, CST } \\
\text { Funcional: HoNOS, VADO. }\end{array}$ & $\begin{array}{l}\text {-Entrenamiento } \\
\text { cognitivo } \\
\text { computarizado } \\
\text { Cogpack. } \\
\text {-Terapias } \\
\text { convencionales. } \\
12 \text { meses }\end{array}$ & $\begin{array}{l}\text { Mejora significativa de las } \\
\text { funciones cognitivas sólo en los } \\
\text { grupos que recibieron el } \\
\text { tratamiento junto a otras terapias, } \\
\text { el entrenamiento cognitivo puede } \\
\text { ser efectivo para las funciones } \\
\text { cognitivas implicadas en } \\
\text { esquizofrenia. }\end{array}$ \\
\hline $\begin{array}{l}\text { Buonocore } \\
\text { et. al. } 2017\end{array}$ & $\begin{array}{l}\text { Esquizofre } \\
\text { nia } \\
\\
60 \\
\text { pacientes }\end{array}$ & $\begin{array}{l}\text { Clínica: PANSS funcion: } \\
\text { Escala de Inteligencia } \\
\text { Adulta } \\
\text { Cognición WechslerR, } \\
\text { Esquizofrenia BACS. }\end{array}$ & $\begin{array}{l}\text {-Entrenamiento } \\
\text { cognitivo } \\
\text { computarizado } \\
\text { Cogpack. } \\
\text {-Terapias } \\
\text { psicológicas y de } \\
\text { entrenamiento } \\
\text { social. } \\
6 \text { meses }\end{array}$ & $\begin{array}{l}\text { Las ganancias obtenidas de la } \\
\text { intervención cognitiva persisten } \\
\text { inclusive a mediano plazo ( } 6 \\
\text { meses y } 5 \text { años), las ganancias } \\
\text { sobre funcionamiento diario } \\
\text { requieren mayores tiempos de } \\
\text { intervención. }\end{array}$ \\
\hline $\begin{array}{l}\text { Bechi, } \\
2017\end{array}$ & $\begin{array}{l}\text { Esquizofre } \\
\text { nia } \\
\\
37 \\
\text { pacientes }\end{array}$ & $\begin{array}{l}\text { Memoria, coordinación } \\
\text { psicomotora, velocidad de } \\
\text { procesamiento, fluidez } \\
\text { verbal, funciones } \\
\text { ejecutivas. } \\
\text { Batería: PANNS, WAIS-R, } \\
\text { BACS, WPS. }\end{array}$ & $\begin{array}{l}\text {-Cogpack Sotfware. } \\
\text {-Terapia laboral y } \\
\text { cognitiva } \\
\text { conductual. } \\
3 \text { meses }\end{array}$ & $\begin{array}{l}\text { El modelo cognitivo mostro } \\
\text { efectos significativos del } \\
\text { coeficiente intelectual y la fluidez } \\
\text { verbal, apoyando el papel clave } \\
\text { de las habilidades neurocognitivas } \\
\text { en el resultado del trabajo, los } \\
\text { resultados no evidenciaron } \\
\text { ninguna influencia de la mejora } \\
\text { cognitiva en el resultado laboral. }\end{array}$ \\
\hline $\begin{array}{l}\text { Katsumi } \\
\text { et. al. } 2017\end{array}$ & $\begin{array}{l}\text { Esquizofre } \\
\quad \text { nia } \\
\\
44 \\
\text { participant } \\
\text { es }\end{array}$ & $\begin{array}{l}\text { Memoria de trabajo, } \\
\text { atención, función ejecutiva. } \\
\text { Batería: BACS, GAF, } \\
\text { JART, PANNS. }\end{array}$ & $\begin{array}{l}\text {-Programa de } \\
\text { rehabilitación } \\
\text { cognitiva utilizando } \\
\text { el software NEAR. } \\
19 \text { sesiones }\end{array}$ & $\begin{array}{l}\text { La memoria verbal, la memoria } \\
\text { de trabajo, la atención y la } \\
\text { función ejecutiva mostraron una } \\
\text { mejora significativamente mayor } \\
\text { en el grupo de intervención } \\
\text { después de la intervención que en } \\
\text { el grupo de control. La función } \\
\text { social y de la vida diaria también } \\
\text { mejoró en el grupo de } \\
\text { intervención y las mejoras se } \\
\text { mantuvieron un año después de la } \\
\text { intervención. }\end{array}$ \\
\hline
\end{tabular}

Fuente: Elaboración propia *incluye los participantes de grupo control. 
Tabla 3.

Resultados

\begin{tabular}{|c|c|c|c|c|}
\hline Autores & $\begin{array}{l}\text { Trastorno y } \\
\text { muestra* }\end{array}$ & $\begin{array}{c}\text { Función abordada } \\
\text { y herramientas }\end{array}$ & $\begin{array}{l}\text { Intervención } \\
\text { y tiempo }\end{array}$ & $\begin{array}{l}\text { Resultado } \\
\text { Relevante }\end{array}$ \\
\hline $\begin{array}{l}\text { Matsuda } \\
\text { et.al. } 2016\end{array}$ & $\begin{array}{l}\text { Esquizofre } \\
\text { nia } \\
62 \\
\text { pacientes }\end{array}$ & $\begin{array}{l}\text { Atención, velocidad } \\
\text { psicomotora, aprendizaje, } \\
\text { memoria y funciones } \\
\text { ejecutivas. } \\
\text { Batería: BACS, PANNS, } \\
\text { JART inteligencia. }\end{array}$ & $\begin{array}{l}\text {-Programa japonés } \\
\text { de rehabilitación } \\
\text { cognitiva para la } \\
\text { esquizofrenia } \\
\text { (JCORES). } \\
12 \text { semanas }\end{array}$ & $\begin{array}{l}\text { El grupo de remediación } \\
\text { cognitiva mostró mejoras } \\
\text { significativamente mayores en la } \\
\text { memoria verbal, la puntuación } \\
\text { compuesta de la Evaluación } \\
\text { breve de la cognición en la } \\
\text { esquizofrenia, la versión } \\
\text { japonesa (BACS-J) y la } \\
\text { psicopatología general en la } \\
\text { Escala de síndrome positivo y } \\
\text { negativo (PANSS). }\end{array}$ \\
\hline $\begin{array}{c}\text { Peña et.al. } \\
2016\end{array}$ & $\begin{array}{l}\text { Esquizofre } \\
\text { nia } \\
111 \\
\text { pacientes }\end{array}$ & $\begin{array}{l}\text { Atención, memoria, } \\
\text { lenguaje, funciones } \\
\text { ejecutivas, planificación } \\
\text { cognitiva y cognición } \\
\text { social. } \\
\text { Función: HVLT, escala de } \\
\text { inteligencia adulta de } \\
\text { WechslerII, test Stroop, } \\
\text { Test Happé } \\
\text { Clinico: PANSS y UCSD }\end{array}$ & $\begin{array}{l}\text {-Programa de } \\
\text { remediación } \\
\text { cognitiva integral } \\
\text { (REHACOP). } \\
4 \text { meses }\end{array}$ & $\begin{array}{l}\text { Se encontró una mejora } \\
\text { significativa en las capacidades } \\
\text { neurocognitivas del grupo tratado } \\
\text { sobre el grupo control, además de } \\
\text { un cambio mayor en cognición } \\
\text { social, no hubo diferencias frente } \\
\text { a sintomatología positiva, sin } \\
\text { embargo, hubo una mayor } \\
\text { mejoría respecto a la } \\
\text { sintomatología negativa. }\end{array}$ \\
\hline $\begin{array}{l}\text { Lanfrediet. } \\
\text { al. } 2016\end{array}$ & $\begin{array}{l}\text { Esquizofre } \\
\text { nia } \\
61 \\
\text { pacientes }\end{array}$ & $\begin{array}{l}\text { Memoria, planificación, } \\
\text { atención visual, habilidades } \\
\text { de control ejecutivo. } \\
\text { Bateria: PANNS, WAIS-R, } \\
\text { HoNOS, Prueba de } \\
\text { aprendizaje verbal de } \\
\text { California. }\end{array}$ & $\begin{array}{l}\text {-Programa Terapia } \\
\text { de rehabilitación } \\
\text { cognitiva }(\mathrm{CRT}) \text {. } \\
50 \text { sesiones }\end{array}$ & $\begin{array}{l}\text { La atención visual se encontró } \\
\text { como el único predictor } \\
\text { significativo de mejora en la } \\
\text { subescala de síntomas negativos } \\
\text { de la Escala de Síndrome Positivo } \\
\text { y Negativo. }\end{array}$ \\
\hline $\begin{array}{l}\text { Fisher et } \\
\text { al, } 2015\end{array}$ & $\begin{array}{l}\text { Esquizofre } \\
\text { nia } \\
86 \text { sujetos }\end{array}$ & $\begin{array}{l}\begin{array}{l}\text { Funciones asociadas al } \\
\text { inicio temprano de } \\
\text { esquizofrenia. }\end{array} \\
\text { Clínica: PANSS } \\
\text { Función: Escala Strauss } \\
\text { Carpenter, MATRICS, D- } \\
\text { KEFS. }\end{array}$ & $\begin{array}{l}\text {-Entrenamiento } \\
\text { cognitivo en } \\
\text { ordenador } \\
\text { controlado a } \\
\text { distancia. } \\
8 \text { semanas }\end{array}$ & $\begin{array}{l}\text { En quienes completaron el } \\
\text { proceso, se encontraron efectos } \\
\text { positivos para el entrenamiento } \\
\text { en la cognición global, la } \\
\text { memoria verbal, y solución de } \\
\text { problemas. Los sujetos se } \\
\text { volvieron más eficientes en el } \\
\text { procesamiento rápido de } \\
\text { estímulos auditivos sucesivos. }\end{array}$ \\
\hline $\begin{array}{c}\text { Garrido } \\
\text { et.al. } 2017\end{array}$ & $\begin{array}{l}\text { Esquizofre } \\
\text { nia } \\
\\
33 \\
\text { participant } \\
\text { es }\end{array}$ & $\begin{array}{l}\text { Velocidad psicomotora, } \\
\text { fluidez fonética, memoria } \\
\text { de trabajo, aprendizaje } \\
\text { verbal, función ejecutiva, } \\
\text { razonamiento y resolución } \\
\text { de problemas. } \\
\text { Funciones: SDMT, FAS, } \\
\text { CVLT, WCST-CV3, Test } \\
\text { Stroop, subpruebas } \\
\text { razonamiento matricial y } \\
\text { secuenciación del WAIS-III }\end{array}$ & $\begin{array}{l}\text {-Remediación } \\
\text { cognitiva asistida } \\
\text { por ordenador } \\
\text { (CACR). } \\
48 \text { sesiones }\end{array}$ & $\begin{array}{l}\text { Se encontró mejora cognitiva } \\
\text { sostenida por un periodo de } 12 \\
\text { meses de las funciones básicas } \\
\text { frecuentemente afectadas en } \\
\text { esquizofrenia como la velocidad } \\
\text { de procesamiento, la memoria de } \\
\text { trabajo, el razonamiento y los } \\
\text { problemas de resolución de los } \\
\text { dominios cognitivos. }\end{array}$ \\
\hline
\end{tabular}

Fuente: Elaboración propia *incluye los participantes de grupo control. 
Tabla 3.

Resultados

\begin{tabular}{|c|c|c|c|c|}
\hline Autores & $\begin{array}{l}\text { Trastorno y } \\
\text { muestra* }\end{array}$ & $\begin{array}{l}\text { Función abordada } \\
\text { y herramientas }\end{array}$ & $\begin{array}{l}\text { Intervención } \\
\text { y tiempo }\end{array}$ & $\begin{array}{l}\text { Resultado } \\
\text { Relevante }\end{array}$ \\
\hline $\begin{array}{l}\text { Harvey, } \\
\text { Balzer, } \\
\text { Kotwicki, } \\
2019\end{array}$ & $\begin{array}{l}\text { Depresión } \\
\text { grave, } \\
\text { trastorno } \\
\text { bipolar y } \\
\text { esquizofre } \\
\text { nia } \\
49 \\
\text { pacientes }\end{array}$ & $\begin{array}{l}\text { Memoria verbal y de } \\
\text { trabajo, velocidad motora, } \\
\text { fluencia semántica. } \\
\text { La Breve Evaluación de la } \\
\text { Cognición para la } \\
\text { Esquizofrenia (BACS). }\end{array}$ & $\begin{array}{l}\text {-Entrenamiento } \\
\text { cognitivo } \\
\text { computarizado } \\
\text { (CCT). } \\
\text { Tiempo variable a la } \\
\text { complitud del } \\
\text { programa. }\end{array}$ & $\begin{array}{l}\text { Los puntajes cognitivos } \\
\text { mejoraron significativamente al } \\
\text { finalizar la intervención, el tipo } \\
\text { de diagnóstico no predice el nivel } \\
\text { de ganancia cognitiva. }\end{array}$ \\
\hline $\begin{array}{l}\text { Tornås, et. } \\
\text { al. } 2017\end{array}$ & $\begin{array}{c}\text { Trastornos } \\
\text { neurodege } \\
\text { nerativos } \\
178 \\
\text { pacientes }\end{array}$ & $\begin{array}{l}\text { Funcionamiento ejecutivo } \\
\text { después de la rehabilitación } \\
\text { cognitiva. } \\
\text { Evaluación de la función } \\
\text { ejecutiva BRIEF-Am } \\
\text { Regulación emocional y } \\
\text { cognición social: BREQ, } \\
\text { BSCQ. }\end{array}$ & $\begin{array}{l}\text {-Entrenamiento de } \\
\text { Manejo de } \\
\text { Objetivos (GMT). } \\
\text {-Psicoeducación }\end{array}$ & $\begin{array}{l}\text { No se encontraron diferencias } \\
\text { significativas entre los grupos al } \\
\text { contar con psicoeducacion. } \\
\text { Mayor CI o edad no implican } \\
\text { factores predictores iniciales para } \\
\text { rehabilitación cognitiva, sin } \\
\text { embargo, si lo hacen en el largo } \\
\text { plazo, mayor CI implica mayor } \\
\text { conciencia de las dificultades } \\
\text { cognitivas y ejecutivas. }\end{array}$ \\
\hline $\begin{array}{l}\text { Mansbach, } \\
\text { Mace y } \\
\text { Clark, } \\
2017\end{array}$ & $\begin{array}{l}\text { Demencia } \\
\text { leve } \\
38 \text { adultos } \\
\text { mayores }\end{array}$ & $\begin{array}{l}\text { Atención y memoria visual. } \\
\text { Función: The Brief } \\
\text { Cognitive Assessment Tool } \\
\text { (BCAT) } \\
\text { Self-rating Inventory of } \\
\text { Cognitive Ability (SRI). }\end{array}$ & $\begin{array}{l}\text {-Programa de } \\
\text { rehabilitación } \\
\text { cognitiva (RC). } \\
\text { Modulo en línea } \\
\text { Memory Match. } \\
9 \text { sesiones en } 3 \\
\text { semanas }\end{array}$ & $\begin{array}{l}\text { Los participantes del grupo de } \\
\text { tratamiento que recibieron el } \\
\text { tratamiento RC obtuvieron } \\
\text { puntuaciones } \\
\text { significativamente más altas } \\
\text { (tamaño de efecto medio a } \\
\text { grande), en la evaluación } \\
\text { posterior, que los participantes } \\
\text { del grupo control durante el } \\
\text { mismo período. }\end{array}$ \\
\hline $\begin{array}{l}\text { Caplain, } \\
\text { Chenuc, } \\
\text { Blancho } \\
\text { Marque, } \\
\text { Aghakhani } \\
\text { (2017) }\end{array}$ & $\begin{array}{l}\text { Traumatis } \\
\text { mo con } \\
\text { patología } \\
\text { psiquiátric } \\
\text { a } \\
\\
97 \\
\text { pacientes }\end{array}$ & $\begin{array}{l}\text { Atención, memoria, control } \\
\text { cognitivo. } \\
\text { Función: MEM III, Escala } \\
\text { de Memoria Wechsler, } \\
\text { Prueba de memoria del } \\
\text { Rey, MINI. }\end{array}$ & $\begin{array}{l}\text {-Manejo } \\
\text { multidimensional } \\
\text { MM: Rehabilitación } \\
\text { cognitiva en } \\
\text { ordenador y } \\
\text { psicoeducación. } \\
6 \text { meses }\end{array}$ & $\begin{array}{l}\text { El rendimiento en el test } \\
\text { neuropsicológico mejoró para los } \\
\text { pacientes con tratamiento } \\
\text { completo, sobre aquellos de } \\
\text { grupo control en los controles } \\
\text { semestral y anual. El pronóstico } \\
\text { también mejoró. }\end{array}$ \\
\hline $\begin{array}{l}\text { Rezapour } \\
(2017)\end{array}$ & $\begin{array}{c}\text { Adicciones } \\
120 \\
\text { pacientes }\end{array}$ & $\begin{array}{l}\text { Aprendizaje, velocidad de } \\
\text { procesamiento, memora de } \\
\text { trabajo. } \\
\text { Prueba psicomotora y de } \\
\text { atención TMT. } \\
\text { Prueba de intervalo de } \\
\text { dígitos (DST). } \\
\text { Prueba Stroop. } \\
\text { Prueba de fluidez verbal } \\
\text { (VFT). Auditory Verbal } \\
\text { Ray Test (RAVLT). }\end{array}$ & $\begin{array}{l}\text { Programa de } \\
\text { rehabilitación } \\
\text { neurocognitiva para } \\
\text { enfermedades de } \\
\text { adicción } \\
\text { (NECOREDA). } \\
8 \text { semanas }\end{array}$ & $\begin{array}{l}\text { Grupo de intervención tuvo un } \\
\text { rendimiento significativamente } \\
\text { mejor en las pruebas de } \\
\text { aprendizaje, cambio, velocidad } \\
\text { de procesamiento, memoria de } \\
\text { trabajo y capacidad de memoria. } \\
\text { Además de un uso de opiáceos } \\
\text { más bajo sobre el grupo de } \\
\text { control durante el seguimiento. } \\
\text { No se observaron diferencias } \\
\text { grupales para la retención del } \\
\text { tratamiento. }\end{array}$ \\
\hline
\end{tabular}

Fuente: Elaboración propia *incluye los participantes de grupo control. 
Tabla 3.

Resultados

\begin{tabular}{|c|c|c|c|c|}
\hline Autores & $\begin{array}{l}\text { Trastorno y } \\
\text { muestra* }\end{array}$ & $\begin{array}{c}\text { Función abordada } \\
\text { y herramientas }\end{array}$ & $\begin{array}{l}\text { Intervención } \\
\text { y tiempo }\end{array}$ & $\begin{array}{l}\text { Resultado } \\
\text { Relevante }\end{array}$ \\
\hline Man 2018 & $\begin{array}{c}90 \\
\text { pacientes }\end{array}$ & $\begin{array}{l}\text { Atención y memoria. } \\
\text { TONi III, habilidades } \\
\text { intelectuales } \\
\text { DVT, atención } \\
\text { RBMT, memoria } \\
\text { WCST, funciones } \\
\text { ejecutivas. }\end{array}$ & $\begin{array}{l}\text { Programa de } \\
\text { entrenamiento } \\
\text { cognitivo } \\
\text { realidad por } \\
(\text { VRG). } \\
15 \text { sesiones }\end{array}$ & $\begin{array}{l}\text { El VRG exhibió mejoras } \\
\text { significativas en la atención y } \\
\text { mejoras en la memoria que se } \\
\text { mantuvieron después de } 3 \text { meses. } \\
\text { Se exhibieron habilidades } \\
\text { vocacionales significativamente } \\
\text { mejoradas después } \\
\text { entrenamiento del } \\
\text { mantuvieron que } \\
\text { seguimiento y durante } \\
\text { autoeficacia. }\end{array}$ \\
\hline $\begin{array}{c}\text { Bell, } \\
\text { Vissicchio } \\
\text { y } \\
\text { Weinstein, } \\
2016\end{array}$ & $\begin{array}{l}\text { Trastorno } \\
\text { por } \\
\text { consumo } \\
\text { de Alcohol } \\
31 \\
\text { participant } \\
\text { es }\end{array}$ & $\begin{array}{l}\text { La tarea de aprendizaje } \\
\text { verbal de Hopkins HVLT; } \\
\text { MINI; ASI; GAF; WTAR } \\
\text { lectura. }\end{array}$ & $\begin{array}{l}\text { Entrenamiento } \\
\text { cognitivo con } \\
\text { software auditivo y } \\
\text { visual Posit Science. } \\
3 \text { meses }\end{array}$ & $\begin{array}{l}\text { Los resultados a los } 3 \text { meses de } \\
\text { seguimiento revelaron efectos } \\
\text { significativos de la condición que } \\
\text { favorecen el entrenamiento } \\
\text { cognitivo para el aprendizaje } \\
\text { verbal. } \\
\text { Hubo una reducción significativa } \\
\text { en el número de participantes en } \\
\text { la condición de entrenamiento } \\
\text { cognitivo con déficits de } \\
\text { memoria verbal clínicamente } \\
\text { significativos. }\end{array}$ \\
\hline $\begin{array}{l}\text { Vita, et al. } \\
2016\end{array}$ & $\begin{array}{l}\text { Trastorno } \\
\text { Límite de } \\
\text { la } \\
\text { Personalid } \\
\text { ad } \\
30 \\
\text { pacientes }\end{array}$ & $\begin{array}{l}\text { Memoria de trabajo y } \\
\text { funcionamiento } \\
\text { psicosocial. } \\
\text { WAIS-R } \\
\text { TMT atención } \\
\text { BACS memoria y función } \\
\text { ejecutiva } \\
\text { SOPT memoria trabajo. }\end{array}$ & $\begin{array}{l}\text { La intervención } \\
\text { CACR: } \\
\text { Entrenamiento } \\
\text { cognitivo } \\
\text { computarizado } \\
\text { CogPack. } \\
16 \text { semanas }\end{array}$ & $\begin{array}{l}\text { Los pacientes del programa } \\
\text { mostraron una mejoría mayor en } \\
\text { la memoria de trabajo y las } \\
\text { medidas de funcionamiento } \\
\text { psicosocial que los pacientes } \\
\text { control. La gravedad de los } \\
\text { síntomas no se vio afectada } \\
\text { significativamente por el } \\
\text { tratamiento con CACR. }\end{array}$ \\
\hline
\end{tabular}

Fuente: Elaboración propia *incluye los participantes de grupo control.

En cuanto a las funciones cognitivas que se trabajaron en la rehabilitación, se encontraban principalmente atención, memoria de trabajo, funciones ejecutivas, aprendizaje verbal, velocidad de procesamiento, velocidad psicomotora, fluidez verbal: semántica y fonológica, así como cognición social. Estas funciones fueron evaluadas mediante un amplio espectro de baterías y pruebas tanto psicológicas como neuropsicológicas, entre las cuales se destacan el Trail
Making Test (TMT) para evaluar funcionamiento motor, Digital Simbol Substitution Test (DSST) que evalúa velocidad de procesamiento de la información, la Escala de inteligencia de Weschler revisada (WAIS-R), que si bien es una prueba de inteligencia, es usada para evaluar funciones cognitivas como habilidades en resolución de problemas y funciones correspondientes al lenguaje, Test de aprendizaje verbal de Hopkins (HVLT), Test de Stroop para valorar la 
atención, Test Happé, para la cognición social, y el Delis-Kaplan Executive Function Systemn (D-KEFS) para evaluar funciones ejecutivas. Estas pruebas fueron aplicadas antes, en un punto medio y luego del proceso de rehabilitación cognitiva.

La rehabilitación cognitiva se administró principalmente mediante la utilización de un software computarizado (9 de los 11 estudios en el área de esquizofrenia utilizaron esta modalidad); el programa más utilizado es el Software CogPack, el cual incluye diferentes ejercicios neurocognitivos que se pueden dividir en dominios, destinados a entrenar áreas cognitivas específicas.

De igual manera, se encontraron estudios exploratorios con la utilización de nuevos programas como es el caso del JCORES, que proporciona ejercicios de práctica en una amplia gama de funciones cognitivas, que incluyen atención, velocidad psicomotora, aprendizaje, memoria y funciones ejecutivas.

Entre los programas de rehabilitación que no incluyen la utilización de un software se encuentra el programa NECOREDA, que consiste en un entrenamiento de las funciones cognitivas mediante actividades en papel y lápiz. Se señala que las funciones que comúnmente se ven afectadas por la esquizofrenia son la atención, memoria, cálculo, proceso visoespacial, habilidades verbales y razonamiento. La duración de estos programas de rehabilitación en los estudios revisados es diversa; por lo general, abarcan procesos de 3 y 6 meses de duración. Además, en algunos estudios, ésta se complementaba con otras modalidades de terapia como farmacológica, ocupacional o grupal.

Los resultados de las investigaciones sugieren que, el grupo que recibió rehabilitación cognitiva, a diferencia de grupo control, presentó mejoría durante el período de seguimiento en funciones cognitivas como la memoria de trabajo y verbal, funciones ejecutivas, cognición social, aprendizaje y fluidez verbal. Esto a su vez implicó mejoría de la funcionalidad social en la vida diaria y se evidenció que estos resultados perduran durante el periodo de seguimiento de $3 \mathrm{y}$ 6 meses; sin embargo, es importante recalcar que los resultados dependieron en gran medida de la gravedad de la sintomatología y de la complementariedad con otras modalidades de tratamiento.

Respecto a los procesos relacionados a otros trastornos, se encontraron dos investigaciones centradas en patologías neurocognitivas además de un estudio que abordaba pacientes con traumatismo y sintomatología psiquiátrica, dentro de los mismos las herramientas de valoración utilizadas evaluaban funciones neurocognitivas básicas con herramientas como el Behavior Rating Inventory of Executive Function for Adults (BRIEF-A), Mini-Mental State Examination (MMSE), Bar Course Aptitud Test (BCAT), además de prestarle particular atención a las funciones ejecutivas y de memoria, pues la alteración de las mismas parece verse asociada a este tipo de trastornos. 
También se examinaron funciones de atención y control cognitivo. La población en estas investigaciones agrupaba edades más avanzadas en relación con las de otras patologías.

Los programas de intervención aplicados en estos estudios fueron diferentes para cada una, pues se direccionaban a partir de las necesidades de cada muestra en particular. Así, un estudio utilizó un software de ordenador para la rehabilitación cognitiva (NEAR). En general se evidenció mejoras en el rendimiento neuropsicológico tras las aplicaciones y por encima de los grupos de control.

$\begin{array}{ccc}\text { Dos procesos integraron } & \\ \text { psicoeducación además de la }\end{array}$ rehabilitación neuropsicológica, aunque no lograron evidenciar diferencias significativas respecto a resultados usando esta modalidad de terapia. Otros resultados señalan que el nivel de coeficiente intelectual (CI) o la edad inicial de los participantes no implican factores que influyan en la rehabilitación cognitiva, aunque sí se evidenció que un mayor CI se relacionaba con mayor consciencia de las dificultades cognitivas y ejecutivas. Finalmente, en pacientes con traumatismos y patología psiquiátrica adicional al avance en rendimiento cognitivo, la prognosis mejoró significativamente para quienes además del tratamiento habitual recibieron rehabilitación cognitiva.

Por otro lado, un grupo de investigaciones se centró en pacientes con patología asociada a las adicciones, con muestras de entre 30 y 120 pacientes, evaluando principalmente rehabilitación de funciones de memoria y atención. En ese sentido, cada investigación diseñó una batería propia para la evaluación cognitiva. Todas las intervenciones ejecutadas utilizaron softwares $y$ procesos diferentes, destacando el uso de un programa de realidad virtual VRG y de un programa de rehabilitación neurocognitiva específico para enfermedades de adicción NECOREDA.

En función de los objetivos de los artículos revisados sobre adicciones, se encontró mejoría en el rendimiento de las funciones cognitivas evaluadas en los procesos de memoria y atención, así como en velocidad del procesamiento, cuyos resultados se mantuvieron durante los procesos de seguimiento posteriores. Uno de los servicios de rehabilitación evidenció una reducción significativa en el número de pacientes que requerían de entrenamiento cognitivo por déficits clínicamente significativos. Los programas se aplicaron en periodos de entre 8 semanas y 3 meses.

Finalmente, sólo un estudio se centró en trastorno de personalidad límite, donde con una muestra de 30 pacientes se buscó intervenir en memoria de trabajo y funcionamiento psicosocial. Tras una intervención de 16 semanas con el programa de entrenamiento cognitivo CogPack, se evidenció una mejoría significativa de las funciones intervenidas sobre aquellas del grupo control; sin embargo, la gravedad sintomatológica propia del trastorno no se vio afectada por el tratamiento. 


\section{Conclusiones}

El objetivo principal de este estudio fue el de indagar acerca de los avances en aplicación de procedimientos de rehabilitación cognitiva en trastornos psiquiátricos, mediante una revisión exhaustiva de la literatura internacional reciente (2015-2020).

Tras el análisis de las investigaciones, se encontró que un gran número de estas giran alrededor del espectro de la esquizofrenia, debido a que este cuadro puede asociarse a alteraciones cognoscitivas especificas (Rodríguez et al., 2019). Además, una cantidad considerable señalan que se aplicaron intervenciones terapéuticas complementarias, sin embargo, la relación existente entre los procesos de rehabilitación cognitiva y otras intervenciones permanece ambigua (Tornas et. al., 2017). Esta ausencia podría sentar un precedente para futuras investigaciones.

En cuanto a la efectividad de lasintervenciones, se encuentra que la mayoría de las investigaciones reportan resultados positivos en función de la rehabilitación cognitiva; una buena parte de los estudios indicó que estos cambios permanecieron evidentes en diversos periodos de tiempo en un rango de evaluaciones posteriores desde 3 meses (Man, 2018) hasta 5 años (Buonocore et. al. 2017), sugiriendo beneficios sostenibles a mediano plazo. En relación con este aspecto, las investigaciones de tipo meta-analítico podrían ayudar a establecer parámetros generales claros en cuestión de efectividad y validez (autor), comprendiendo que desde una perspectiva estadística la mayoría de los estudios manejan muestras pequeñas.

Los modelos de intervención en su mayoría se dieron a través de programas computarizados, ante esto se debe recalcar que algunos de los estudios se diseñaron como exploratorios para estas herramientas (Mansbach, Mace y Clark, 2017; Matsuda et.al. 2016) mientras otros representaron adaptaciones para condiciones particulares (Buonocore et. al. 2017; Katsumi et. al. 2017; Vita, et al. 2016), y desde este ámbito, se sugiere que la investigación sobre las mismas tenga un mayor refinamiento, pues aparentan tener un potencial diverso en su uso.

Finalmente, a más de la efectividad comprobada para rehabilitación cognitiva, se debe rescatar la noción de un tratamiento integral e interdisciplinario, tanto en investigación como en la aplicación para la clínica diaria, puesto que, desde la literatura revisada, algunos autores toman en cuenta la farmacoterapia (Katsumi et. al. 2017; Rezapour et al., 2017), la terapia psicológica (Corrivetti, Caserta, Perna, 2018; Harvey, Balzer, Kotwicki, 2019; Rodríguez et al., 2019), y terapias ocupacionales (Bell, Vissicchio y Weinstein, 2016). Existen indicios de que las intervenciones combinadas implican también beneficios adicionales dentro de la sintomatología (Peña et al., 2016) y funcionalidad social (Vita, et al., 2016) en los pacientes. De esta manera se puede destacar cómo la rehabilitación cognitiva debe estar siempre de la mano de las llamadas terapias convencionales en el 
tratamiento de trastornos psiquiátricos, en aras de un mejoramiento integral en la condición de los pacientes.

La principal limitación fue que gran parte de los estudios no mostraron de forma clara cómo se ejecutó el proceso de rehabilitación específico y el tipo de modalidad que siguió en relación con el grupo experimental y grupo control, por lo que se decidió dejar de lado este aspecto al momento de considerar los resultados. Otro aspecto para tomar en consideración es que la mayoría de literatura revisada se origina fuera de Latinoamérica, esta carencia implica también la necesidad de desarrollar herramientas propias, así como de adaptar las disponibles.

\section{Referencias}

Bechi, M., Spangaro, M., Pigoni, A., Ripamonti, E., Buonocore, M., Cocchi, F., Bianchi, L., Guglielmino, C., Mastromatteo, A., Cavallaro, R., \& Bosia, M. (2017). Exploring predictors of work competence in schizophrenia: The role of theory of mind. Neuropsychological Rehabilitation, 29(5), 691-703. http://dx.doi.org/10. 1080/09602011.2017.1314217

Bell, M., Vissicchio, N., \& Weinstein, A. (2016). Cognitive Training and Work Therapy for the Treatment of Verbal Learning and Memory Deficits in Veterans with Alcohol Use Disorders. Journal of Dual Diagnosis, 12(1), 83-89. h t t p : / / d x . d o i . o r g / 10.1080/15504263.2016.1145779

Buonocore, M., Spangaro, M., Bechi, M., Baraldi, M. A., Cocchi, F., Guglielmino, C., Cavallaro, R. (2018). Integrated cognitive remediation and standard rehabilitation therapy in patients of schizophrenia: persistence after 5 years. Schizophrenia Research, 192, 335-339. http://dx.doi. org/10.1016/j.schres.2017.05.022

Caplain, S., Chenuc, G., Blancho, S., Marque, S. y Aghakhani, N. (2019). Efficacy of Psychoeducation and Cognitive Rehabilitation After Mild Traumatic Brain Injury for Preventing Post-concussional Syndrome in Individuals With High Risk of Poor Prognosis: A Randomized Clinical Trial. Frontiers in Neurology. http://dx.doi.org/ 10.929.10.3389/fneur.2019.00929.

Cardoner, N., \& Vicent, M. (2017). Rehabilitación cognitiva en salud mental. Sobre ruedas, (95), 16-19.

Casas, E., Escandell, M. J., Ribas, M., \& Ochoa, S. (2010). Instrumentos de evaluación en rehabilitación psicosocial. Revista de la Asociación Española de Neuropsiquiatría, 30(1), 25-47.

Corrivetti, G. \& Caserta, D. \& Perna, C. (2018). Implementation of cognitive rehabilitation in psychiatric services: Integration of cognitive remediation and psychosocial rehabilitation. Journal of Psychopathology. 24, 93-97.

Cuesta, M. J., Moreno-Izco, L., Ribeiro, M., LópezIlundain, J. M., Lecumberri, P., Cabada, T., Peralta, V. (2018). Motor abnormalities and cognitive impairment in first-episode psychosis patients, their unaffected siblings and healthy controls. Schizophrenia research, 200, 50-55.

Cuesta, M. J., Sánchez-Torres, A. M., Cabrera, B., Bioque, M., Merchán-Naranjo, J., Corripio, I., Sanjuan, J. (2015). Premorbid adjustment and clinical correlates of cognitive impairment in first-episode psychosis. Schizophrenia Research, 164(1-3), 65-73.

Garcelan, S. P. (2015). La necesidad de la rehabilitación psicosocial en el tratamiento de la 
esquizofrenia. Información psicologica, (83), 37-46.

Garrido, G., Penadés, R., Barrios, M., Aragay, N., Ramos, I., Vallès, V., ... Vendrell, J. M. (2017). Computer-assisted cognitive remediation therapy in schizophrenia: Durability of the effects and cost-utility analysis. Psychiatry Research, 254, 198 204.http://dx.doi.org/10.1016/j.psychres. 2017.04.065

Fisher, M., Loewy, R., Carter, C., Lee, A., Ragland, J. D., Niendam, T., ... Vinogradov, S. (2014). Neuroplasticity-Based Auditory Training Via Laptop Computer Improves Cognition in Young Individuals With Recent Onset Schizophrenia. Schizophrenia Bulletin, 41(1), 250-258. http://dx.doi.org/10.1093/schbul/sbt232

Harvey, P. D., Balzer, A. M., \& Kotwicki, R. J. (2019). Training engagement, baseline cognitive functioning, and cognitive gains with computerized cognitive training: A cross-diagnostic study. Schizophrenia Research: Cognition. http://dx.doi.org/10.1016/j.scog.2019.10 0150

Katsumi, A., Hoshino, H., Fujimoto, S., Yabe, H., Ikebuchi, E., Nakagome K., \& Niwa, S. (2017). Effects of cognitive remediation on cognitive and social functions in individuals with schizophrenia. Neuropsychological Rehabilitation, 29(9), 1475-1487. http://dx.doi.org/10. 1080/09602011.2017.1409639

Lanfredi, M., Deste, D., Ferrari, C., Barlati, S., Magni, L., Rossi, R., Peri, L., Bonomi, M., Rossi, G., \& Vita, A. (2016). Effects of cognitive remediation therapy on neurocognition and negative symptoms in schizophrenia: an Italian naturalistic study. Cognitive Neuropsychiatry, 22(1), 53-68. http://dx.doi.org/10.1080/135468 05.2016 .1260537

Man, D. (2018) Virtual reality-based cognitive training for drug abusers: A randomised controlled trial. Neuropsychological Rehabilitation, 30(2), 315-332. http://dx. doi.org/10.1080/09602011.2018.1468271

Mansbach, W., Mace R., \& Clark, K. (2017). The Efficacy of a Computer-Assisted Cognitive Rehabilitation Program for Patients with MildCognitive Deficits: A Pilot Study. Experimental Aging Research, 43(1), 94-104. http://dx.doi. org/10.1080/0361073X.2017.1258256

Matsuda, Y., Morimoto, T., Furukawa, S., Sato, S., Hatsuse, N., Iwata, K., Kimura, M., Kishimoto, T., \& Ikebuchi, E. (2016). Feasibility and effectiveness of a cognitive remediation programme with original computerised cognitive training and group intervention for schizophrenia: a multicentre randomised trial. Neuropsychological Rehabilitation, 28(3), 387-397. http://dx.doi.org/10. 1080/09602011.2016.1181555

Moher, D., Liberati, A., Tetzlaff, J., \& Altman, D. G.(2009). Preferred Reporting Items for Systematic Reviews and Meta-Analyses: The PRISMA Statement. PLoS Medicine, $6(7)$.

Moreno, J. J. P., García, M. R., Gámez, A. S., \& Moreno, B. O. (2016). Estudio piloto. Memoria implícita, memoria explícita y deterioro cognitivo: evolución en el trastorno psicótico. Enfermería Global, 15(1), 135-152.

Ojeda, N., Peña, J., Bengoetxea, E., García, A., Sánchez, P., Segarra, R.,... \& Eguíluz, J. I. (2012). REHACOP: programa de rehabilitación cognitiva en psicosis. Rev Neurol, 54. 3-42.

Peña, J., Ibarretxe-Bilbao, N., Sánchez, P., Iriarte, M. B., Elizagarate, E., Garay, M. A., Ojeda, N. (2016). Combining social cognitive treatment, cognitive remediation, and functional skills training in schizophrenia: a randomized controlled 
trial. Npj Schizophrenia, 2(1).

http://dx.doi.org/10.1038/npjschz.2016.37

Perona, S. (2003). La necesidad de la rehabilitación psicosocial en el tratamiento de la esquizofrenia. 83. 37-46.

Rezapour, T., Hatami, J., Farhoudian, A., Sofuoglu, M., Noroozi, A., Daneshmand, R., Samiei, A., \& Ekhtiari, H. (2017). Cognitive rehabilitation for individuals with opioid use disorder: A randomized controlled trial. Neuropsychological Rehabilitation, 29(8), 1273-1289. http://dx.doi.org/10.1080/09602011.2017 .1391103 